Article

\title{
A Study into the Effects of Factors Influencing an Underwater, Single-Pixel Imaging System's Performance
}

\author{
Qi Chen ${ }^{1,2}$, Anumol Mathai ${ }^{1}$, Xiping $\mathrm{Xu}^{2}$ and Xin Wang ${ }^{1, *(\mathbb{C}}$ \\ 1 School of Engineering, Monash University Malaysia, Jalan Lagoon Selatan, Bandar Sunway 47500, Malaysia; \\ 2014100211@mails.cust.edu.cn (Q.C.); anumol.mathai@monash.edu (A.M.) \\ 2 College of Optoelectronic Engineering, Changchun University of Science and Technology, Changchun \\ 130022, China; xxp@cust.edu.cn \\ * Correspondence: wang.xin@monash.edu
}

Received: 20 September 2019; Accepted: 5 November 2019; Published: 27 November 2019

\begin{abstract}
Underwater detection has always been a challenge due to the limitations caused by scattering and absorption in the underwater environment. Because of their great penetration abilities, lasers have become the most suitable technology for underwater detection. In all underwater laser applications, the reflected laser pulse which contains the key information for most of the system is highly degraded along the laser's propagation path and during reflection. This has a direct impact on the system's performance, especially for single-pixel imaging (SPI) which is very dependent on light-intensity information. Due to the complications in the underwater environment, it is necessary to study the influential factors and their impacts on underwater SPI. In this study, we investigated the influence of the angle of incidence, target distance, and medium attenuation. A systematic investigation of the influential factors on the reflectance and ranging accuracy was performed theoretically and experimentally. The theoretical analysis was demonstrated based on the bidirectional reflection distribution function (BRDF) and laser detection and ranging (LADAR) model. Moreover, 2D single-pixel imaging (SPI) systems were setup for experimental investigation. The experimental results agree well with the theoretical results, which show the system's dependency on the reflection intensity caused by the angle of incidence, target distance, and medium attenuation. The findings should be a reference for works looking to improve the performance of an underwater SPI system.
\end{abstract}

Keywords: laser sensing; BRDF; LADAR; reflection; absorption; single pixel imaging

\section{Introduction}

Over the past decade, various investigations have aimed to detect or image an object in an underwater environment [1-5]. Lasers have been employed in various fields, such as reverse engineering, machine vision, 3D imaging, and especially, underwater object detection [6-9]. It became a suitable choice for applications like optical metrology [10], target recognition [11], surface profiling [12], object modeling [13], and 3D vision [14] because of its monochromaticity, collimation, and penetrability properties. Several techniques have been used for underwater object detection, such as range-gated [15,16], modulated LIDAR [17,18], and streak-tube imaging [19]. During the light propagation and the interaction with the target, numerous factors can affect the reflected laser pulse, such as laser source, target surfaces, detection unit, and transmittance medium [20,21]. These are important, especially in underwater environment because target surface optical characteristics and light transmittance of the medium are complex and volatile. In laser systems, the key information 
is extracted from the reflected laser pulse, which directly affects the system performance. Tan et al. analyzed the effect of scattering on the three-demonstration (3D) machine vision by using a gated imaging system [20]. Chua et al. studied the influence of interference on distance and derived a new $3 \mathrm{D}$ range gated model [22]. Wang et al. investigated the influence of a laser's profile on a system's accuracy [23,24]. In addition, many researchers have investigated the influence of setup parameters on system performance. The sensor parameters' effects and system performance are discussed in [25,26]. Laux et al. used an optical vortex as a spatial coherence analyzer in order to increase accuracy of range in underwater pulsed laser ranging system [27]. Zheng et al. demonstrated a high-speed and high-resolution experimental setup to detect objects in a turbid underwater environment by using particular laser sources [28-30]. A beamforming receiver and near-field beamforming algorithm were developed ratio to minimize scattering effect and improve 3D image reconstruction performance in water medium by Walter et al. [31]. Moreover, Duan et al. present a quantitative calculation model to accurately analyze the signal-to-noise ratio (SNR) in fog by using the Mie theory [32]. Le et al. indicates that computational imaging has the advantages of a disturbance-free and wider angle of view (AOV), which can effectively improve the imaging result [3]. In addition, as a representative of computational imaging, single-pixel imaging (SPI) has been applied in environments where the background light is extremely dark or even occluded [33]. Therefore, it is essential to study the transmission characteristics of a laser under environmental influence and the influence on the quality of an SPI system. Remarkably, SPI can solve this problem by keeping the object beam and exploiting calculated field pattern rather than reference beam. Although the setup is simplified, the advantage of SPI, i.e., being disturbance-free, remains.

However, there are limited studies of the influence of the angle of incidence, target distance, and medium on a reflectance system's performance and a computational imaging system's quality. Therefore, it is necessary to investigate the influence of these factors, which will directly impact the system's performance. Based on bidirectional reflection distribution function (BRDF) and laser detection and ranging (LADAR) models, we investigated the reflectance characteristics, combing both microsurface and macrosurface theories. It is well known that most of the underwater laser-based object-detection techniques obtain valuable data by employing peak detection which is a reliable and handy technique for ranging [34,35]. In order to reduce effect of noise from back scattering light, we proposed a simple and stable, pulsed SPI system, and employed peak detection to conduct an experimental study.

We begin with a brief introduction of BRDF and LADAR model in Section 2. In Section 3, we show the relationship between the influential factors of the angle of incidence, target distance, and medium attenuation on the reflectance by demonstrating a theoretical derivation based on BRDF and LADAR model with the microsurface and macrosurface theories. Furthermore, the effects of these influential factors on the reflectance and system performance are analyzed theoretically. In our experimental investigation, an underwater, active-mode SPI was built to determine the reflection ratio and intensity error. This is further explained in Section 4. The experimental results obtained are discussed in Section 5. At last, we conclude our findings in Section 6.

\section{Background}

\subsection{SPI Theory}

SPI system works by gathering bucket sums of a light field which has interacted with an object under investigation, and a spatial light modulator. Standard SPI system architecture consists of following the main components: the light source, optics, single pixel detector, and spatial light modulator (SLM). A series of modulator-generated patterns are illuminated the object by using SLM and the corresponding intensity value of each pattern is collected by single-pixel photodetector, which is only sensitive to the intensity but not the phase of electromagnetic radiation. 
According to the definition, the patterns generated by the modulator (DMD (digital micromirror device)) illuminate the object and result in the circulation of the intensity function $Y$ that contains the product of corresponding $X$ and $\boldsymbol{\Phi}$ values at each pixel location. $M$-dimensional column vector $Y$ and projected illuminating patterns can be concluded in terms of a single matrix equation:

$$
Y_{M \times 1}=\boldsymbol{\Phi}_{M \times N} \times X_{N \times 1}
$$

in which, $M$ represents the number of measurements, $N$ is dimension of original signal, and $\boldsymbol{\Phi}$ is the whole measurement with dimension $M \times N$. Classical matrix inversion cannot solve the unique solution of the Equation (1), which is under-determined in nature. However, when the measurement matrix $(\boldsymbol{\Phi})$ satisfies the restricted isometry property (RIP) and sparse signal, $(X)$ is $\mathrm{K}$-sparse; then, the signal can be recovered by employing $l_{1}$ norm minimization problem with large probability and linear programming [36]. Practically, many signals do not exist with sparsity in their original form but can be represented sparsely on some suitable basis.

The underwater object detection and image acquisition process we propose are shown in Figure 1. The signal strength of the object that has to be imaged was set as $N$ which represents the total number of pixels in our reconstructed image. For the following step, the number of the observations $(m)$ needed for reconstruction is calculated based on $m=O(K \log N)$. The sparsity of the binary matrix is important in the next step to limit the number of observations to $m$. The experiment will run until the number of measurements is equal to the number of observations. Then, $l_{1}$ minimization algorithm does the initial guess of the original signal $X$. This initial value is updated after each iteration of the $l_{1}$ minimization algorithm. The result is displayed in the final step.

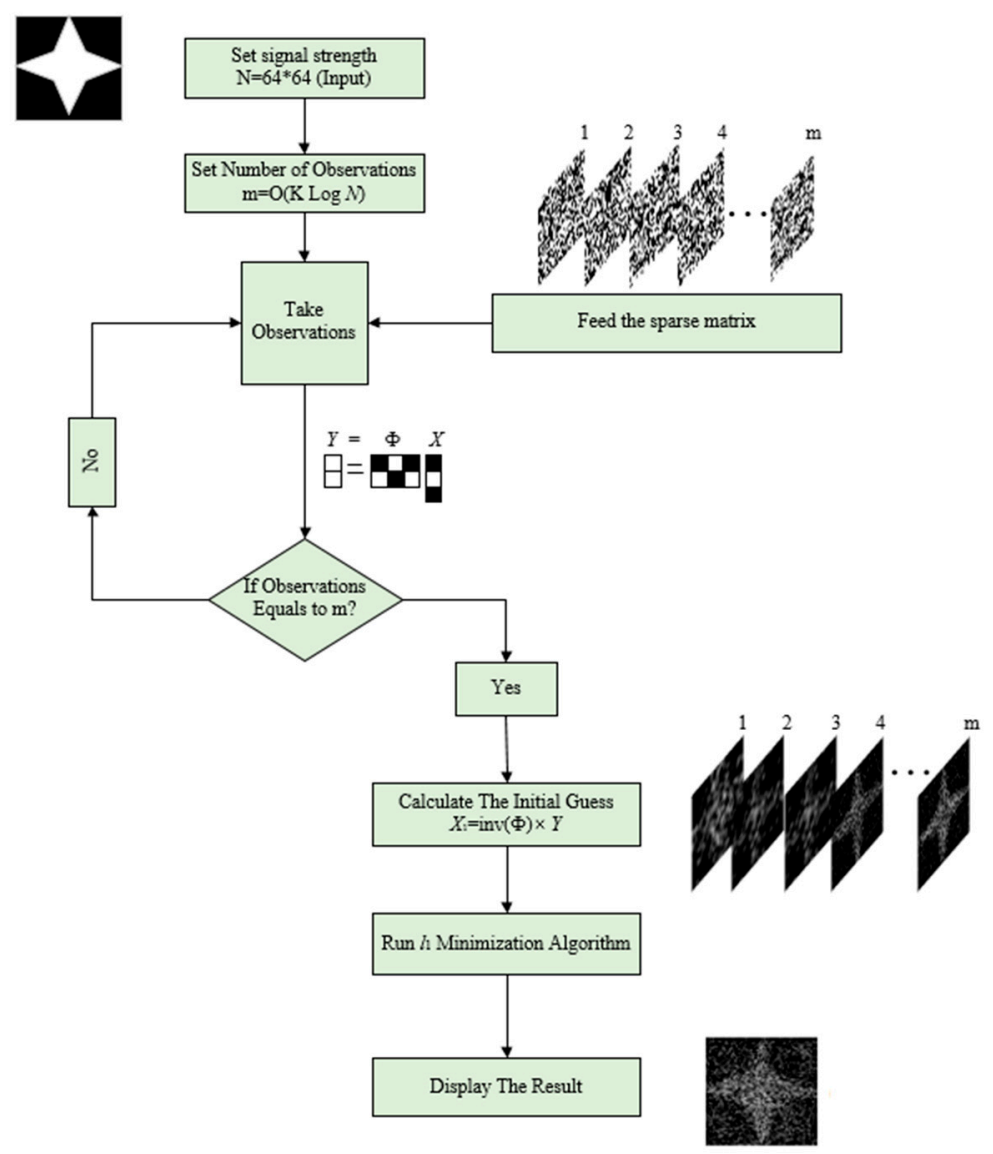

Figure 1. Flow chat of the approach for underwater single-pixel imagine (SPI). 
Based on the above definition of SPI and its image reconstruction characteristics, it can be clearly observed that the reconstruction imaging quality of a SPI system is closely related to the intensity of light reflected from an object. Because lasers have good coherence characteristics, and SPI systems are less affected by optical coherence characteristics, SPI systems relying on lasers as the light source are most common. However, the underwater environment is very complicated, and the reflected light intensity after being adjusted will be affected by various factors in the environment. So, it is very essential to study the transmission and reflection characteristics of the reflected light intensity in the underwater environment and the impact on the system.

\subsection{LADAR Model}

Typically, each echo laser energy in an SPI system is influenced by multiple factors can be given by the LADAR equation [37]:

$$
Y_{m}=P_{R}=P_{T} \frac{\theta_{R}^{2}}{\theta_{T}^{2}} \frac{\rho_{T}}{\pi} \cos \theta_{t} \frac{A_{R}}{R^{2}} \eta_{T} \eta_{R} \eta_{A}^{2}
$$

where $P_{T}, P_{R}\left(Y_{m}\right)$, and $R$ are the emitted energy, received energy, and range across, respectively; $Y_{m}$ is the $m$ th measurement value; $\theta_{R}$ is the field of view of the receiver; $\theta_{T}$ is the divergence angle of the laser beam; $\rho_{T}$ corresponds to the target reflectivity; $\theta_{t}$ is the angle of incidence of the laser beam relative to the macroscopic surface normal direction; $A_{R}$ is the area of the aperture of the receiver; $\eta_{T}$, $\eta_{R}$, and $\eta_{A}$, are the loss factors due to beam shaping unit inefficiency, receive system inefficiency, and one-way water attenuation caused by scattering and absorption, respectively. It can be seen that $\rho_{T}$ is attributed to the target reflection characteristics; the strength of the echo signal will change with the change of the target, which will lead to further changes in measurement accuracy.

\subsection{BRDF Reflection Model}

Reflection occurs when the laser pulse hits the interface between two dissimilar media; for instance, water and the target. This relates to aforementioned parameters: target reflectivity $\rho_{T}$, which quantifies the reflective nature of the target. The BRDF is a function of four real variables that defines how light is reflected at an optics surface.

The BRDF was first defined by Fred Nicodemus [38] around 1965. The definition is:

$$
\rho_{T}=f_{r}(l, v)=\frac{d L(v)}{d E(l)}
$$

where $l$ is light direction and $v$ is the view direction.

Physically, with these assumptions (locally optically flat surface, no interreflections), a BRDF term can be derived from Equation (4) $[39,40]$. This BRDF term has the following form:

$$
\rho_{T}=f_{b}+f_{s}
$$

\section{Theoretical Investigation}

Using LADAR model and BRDF reflection model, a relationship between the reflection intensity and the intensity error with the target reflection is established to extract parameters target material, color, and surface roughness involved in the BRDF model for characterization of the target. Based on Equations (1) and (3), we get:

$$
P_{R}=P_{T} \frac{\cos \theta_{t} \theta_{R}^{2} A_{R} \eta_{T} \eta_{R}}{\pi \theta_{T}^{2}}\left(f_{b}+f_{s}\right) \frac{1}{R^{2}} \eta_{A}^{2}
$$


In Equation (4), diffusion and specular reflection was given by O. Steinvall [41]:

$$
\begin{gathered}
f_{b}=K_{D} \cos ^{m} \theta \\
f_{s}=\frac{K_{S}}{\cos ^{6} \theta} \exp \left(\frac{-\tan ^{2} \theta}{s^{2}}\right)
\end{gathered}
$$

where $\theta$ is the incident and reflected angle, $s$ is the surface slope, and $m$ is the diffusivity coefficient. $K_{D}$ is the diffuse reflection constant and $K_{S}$ is the constant of specular reflection, and the relationship between the two parameters $\frac{K_{D}}{K_{S}}$ designates the ratio of diffuse performance to surface glint behavior.

Combining Equations (4)-(6) yields:

$$
P_{R}=P_{T} \frac{\cos \theta_{t} \theta_{R}^{2} A_{R} \eta_{T} \eta_{R}}{\pi \theta_{T}^{2}}\left(K_{D} \cos ^{m} \theta+\frac{K_{S}}{\cos ^{6} \theta} \exp \left(\frac{-\tan ^{2} \theta}{s^{2}}\right)\right) \frac{1}{R^{2}} \eta_{A}^{2}
$$

Equation (8) illuminates that reflected laser pulse intensity $P_{R}$ is influenced by multiple factors, including laser, detector, target, and atmospheric parameters. When target and system are fixed the reflected laser-pulse intensity only depends on the influential factors, such as angle of incidence, target distance, and medium attenuation.

Achievable accuracy $\sigma_{R}$ for the $Y_{m}$ which is based on the peak detection algorithm with a pulse width of $\tau_{\text {laser }}(\mathrm{FWHM})$ can be estimated by the following (reported in [42-45]).

$$
\begin{aligned}
\sigma_{R} & =0.6 \frac{c_{w} \tau_{\text {laser }}}{S N R} \\
S N R & =\frac{\text { Signal Energy }}{\text { Noise Energy }}
\end{aligned}
$$

where $c_{w}$ is the speed of light and $\tau_{\text {laser }}$ is the bandwidth of laser pulse; i.e., the full width at half maximum (FWHM)of the laser pulse profile. In order to keep the noise energy constant and avoid the influence of other factors, the other factors are kept unchanged when studying a specific factor so that the SNR is the signal-to-noise ratio for a single parameter. In our investigation, semi-quantitative analysis was employed as our analysis method, which is often used when quantitative analysis is very difficult. Therefore, noise energy in our research was the sum of the detector's electronic noise and ambient noise, which remained constant throughout the experiment.

In the pulsed laser-ranging system, the signal energy is equal to reflected pulse-laser energy $P_{R}$ which means $P_{R}=$ Signal Energy. Then, Equation (9) can be resolved to obtain SNR as:

$$
S N R=\frac{P_{R}}{\text { Noise Energy }}
$$

combining Equation (9) with Equation (10) $\sigma_{R}$ can be written as:

$$
\sigma_{R}=0.6 \frac{c_{w} \tau_{\text {laser }} \text { Noise Energy }}{P_{R}}
$$

Substitute Equation (7) into Equation (11). We get:

$$
\sigma_{R}=0.6 \frac{\pi c_{w} \tau_{\text {laser }} \text { Noise Energy } \theta_{T}^{2}}{P_{T} \cos \theta_{t} \theta_{R}^{2} A_{R} \eta_{T} \eta_{R}} \frac{R^{2}}{\eta_{A}^{2}} \frac{1}{K_{D} \cos ^{m} \theta+\frac{K_{S}}{\cos ^{6} \theta} \exp \left(\frac{-\tan ^{2} \theta}{s^{2}}\right)}
$$

It can be seen from Equation (12), that the intensity accuracy has strong dependency on target distance, attenuation coefficient, and the angle of incidence. The effect of these parameters on the intensity accuracy is analyzed theoretically and experimentally in this paper. 


\subsection{Influence of Angle of Incidence}

Incidence angle is the angle between a ray incident on a surface and the line perpendicular to the surface at the point of incidence, called the normal. The angle of incidence can be characterized by using a suitable BRDF, whose definition is shown in Equations (2) and (3). Due to complicated scenes and geometry, for example, regarding its edges and shape, the angle of incidence cannot ever be zero. Therefore, it is necessary to know the effect of angle of incidence variation and its effect on reflectance and intensity accuracy. The BRDF model used in this paper consists of specular and diffuse reflections. Using the BRDF function which is shown Equations (3), (5) and (6), we studied the influence of the angle of incidence on the reflection ratio of the system theoretically. Figures 2-4 are simulation results of BRDF functions showing the reflective nature of different parameters which are outlined in legends at the right side of each of simulated plots (1)-(3).

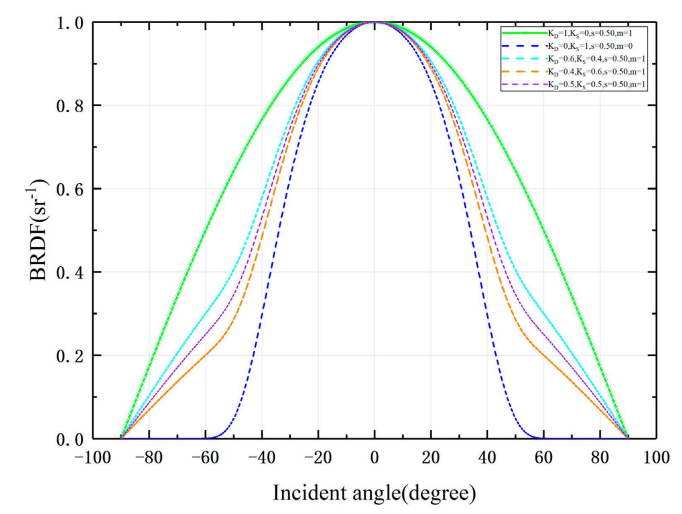

Figure 2. Bidirectional reflection distribution function (BRDF) as a function of various values of diffuse and specular constants taken from Table 1.

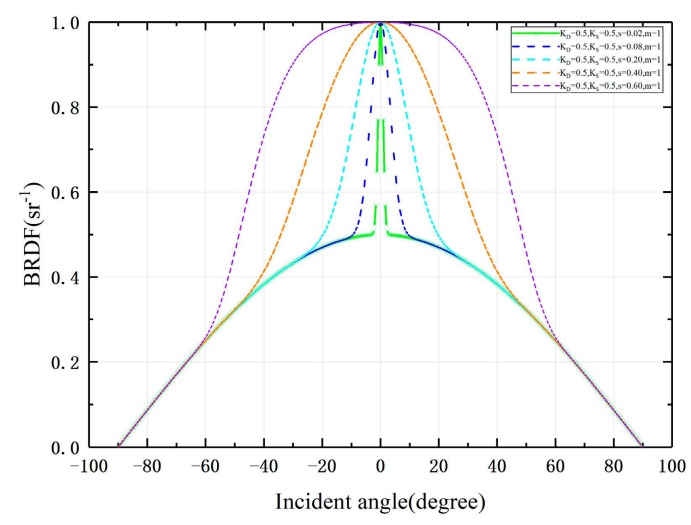

Figure 3. BRDF as a function of various diffuse and specular constants taken from Table 2. 


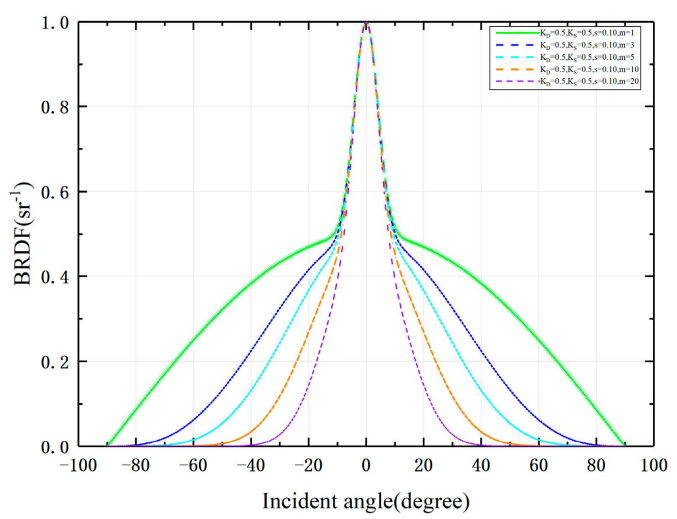

Figure 4. BRDF as a function of various values of diffuse and specular constants taken from Table 3.

Table 1. Different parameters of BRDF used to analyze the effect of the angle of incidence.

\begin{tabular}{ccccc}
\hline Target Reflectance Characteristic & $\boldsymbol{K}_{\boldsymbol{D}}$ & $\boldsymbol{K}_{\boldsymbol{S}}$ & $\boldsymbol{s}$ & $\boldsymbol{m}$ \\
\hline Completely rough & 1 & 0 & 0.5 & 0 \\
Completely smooth & 0 & 1 & 0.5 & 1 \\
$K_{D}>K_{S}$ & 0.6 & 0.4 & 0.5 & 1 \\
$K_{D}<K_{S}$ & 0.4 & 0.6 & 0.5 & 1 \\
$K_{D}=K_{S}$ & 0.5 & 0.5 & 0.5 & 1 \\
\hline
\end{tabular}

Firstly, we must keep the surface slop and diffuse coefficient constant and choose different $K_{D}$ and $K_{S}$ values. Referring Figure 2 and Table 1, it can be seen that reflection taking place in a small span of angle where error is noted at around $60^{\circ}$, is case of pure specular reflection. In case of pure diffuse reflection from a surface, it is in all the directions and intensity error is anticipated at around $90^{\circ}$. We can see that with the increase of an angle of incidence in each target model, the BRDF values decrease, which means the reflected laser pulse intensity decreases at the same time. As the angle increases, the value of BRDF drops down very fast, which means the intensity error is high because only a small change in the angle of incidence would give a high impact. Hence, our observation indicates that it is highly recommended to keep the angle of incidence as small as possible, while performing intensity data experiments.

Secondly, we must choose different surface slope values and keep the other factors constant. Figure 3 and Table 2 show the relationship between BRDF and the angle of incidence when we change the surface slope. Surface slope is a factor relying on the surface roughness; the higher the surface roughness, the higher the value of $s$. We can easily see that with an increasing angle of incidence, the BRDF value goes down. For the maximum value of surface slope $s=0.60$, from Table 2, the roughness is highest for this surface among all five and the value of BRDF falls down most slowly because of the high diffusion intensity. When we increase the angle of incidence more than $60^{\circ}$, the value for the BRDF goes down for all the samples with almost same trend because of the shadowing and masking effects which are almost the same for the five sample surfaces.

Table 2. Different parameters of BRDF used to analyze the surface slope.

\begin{tabular}{ccccc}
\hline Surface Number & $\boldsymbol{K}_{\boldsymbol{D}}$ & $\boldsymbol{K}_{\boldsymbol{S}}$ & $\boldsymbol{s}$ & $\boldsymbol{m}$ \\
\hline 1 & 0.5 & 0.5 & 0.02 & 1 \\
2 & 0.5 & 0.5 & 0.08 & 1 \\
3 & 0.5 & 0.5 & 0.20 & 1 \\
4 & 0.5 & 0.5 & 0.40 & 1 \\
5 & 0.5 & 0.5 & 0.60 & 1 \\
\hline
\end{tabular}


In the end, we chose five different surfaces with different values of diffusivity coefficient, as shown in Table 3. According to Figure 4, it can be seen that when the angle of incidence equal to $0^{\circ}$ the BRDF attains its highest value. The value of BRDF goes down drastically when the angle of incidence is below $20^{\circ}$ and after $20^{\circ}$ the slow variation in BRDF function can be noted. The width of curves become shorter and shorter when the attenuation coefficient increases, which prohibits the light to coming out due to the shadowing and masking problem.

Table 3. Different parameters of BRDF used to analyze the effect of angle of incidence.

\begin{tabular}{ccccc}
\hline Surface Number & $\boldsymbol{K}_{\boldsymbol{D}}$ & $\boldsymbol{K}_{\boldsymbol{S}}$ & $\boldsymbol{s}$ & $\boldsymbol{m}$ \\
\hline 1 & 0.5 & 0.5 & 0.1 & 1 \\
2 & 0.5 & 0.5 & 0.1 & 3 \\
3 & 0.5 & 0.5 & 0.1 & 5 \\
4 & 0.5 & 0.5 & 0.1 & 10 \\
5 & 0.5 & 0.5 & 0.1 & 20 \\
\hline
\end{tabular}

The above plots of BRDF match the experimental data obtained alongside Michael Ashikhmin [46]. Both the simulation's BRDF value and experimental measurements of reflected pulse intensity decrease with an increase in angle of incidence. As a result, intensity error increases, which is proved in Equation (12).

\subsection{Influence of Target Distance and Medium Attenuation}

From Equation (12) it can be observed that the intensity accuracy of pulse-laser ranging system also depends on both target distance and medium attenuation caused by absorption and scattering. That means both target distance and intensity error are directly proportional. The loss factor $\eta_{A}$ can be written as:

$$
\eta_{A}=T(R)=\exp (-c R)
$$

in which $R$ is the target distance and $c$ is the attenuation coefficient of the target medium. Substituting Equation (13) into Equation (12), we can get:

$$
\sigma_{R}=0.6 \frac{\pi c \tau_{\text {laser }} \text { Noise Energy } \theta_{T}^{2}}{P_{T} \cos \theta_{t} \theta_{R}^{2} A_{R} \eta_{T} \eta_{R}} \frac{1}{K_{D} \cos ^{m} \theta+\frac{K_{S}}{\cos ^{6} \theta} \exp \left(\frac{-\tan ^{2} \theta}{s^{2}}\right) \frac{\exp ^{2}(-c R)}{R^{2}}}
$$

The two parameters $R$ and $c$ are considered together, as target distance also affects the value of the loss factor. Exponential $A$ is defined as the impact factor of intensity error, which contains these two parameters:

$$
A=\frac{\eta_{A}^{2}}{R^{2}}=\frac{\exp ^{2}(-c R)}{R^{2}}
$$

From Equations (4) and (15), it can be seen that the higher the value of impact factor is, the more pulse energy that would be reflected, and the smaller the impact factor, the larger the error would be, corresponding to Equation (14). Firstly, the impact factor changes with the increase in distance while keeping $c$ constant. Figure 5 illustrates the diverse values of $c$ ranging from $0.2 \mathrm{~m}^{-1}$ to $1.2 \mathrm{~m}^{-1}$. Based on Figure 5 we can conclude that the impact factor goes down with increasing distance. Decrements of the impact factor can be clearly seen when $c$ goes down and $R$ is constant, which means that the effect of $c$ on impact factor is inversely proportional. Ultimately intensity error increases with the decay of impact factor. 


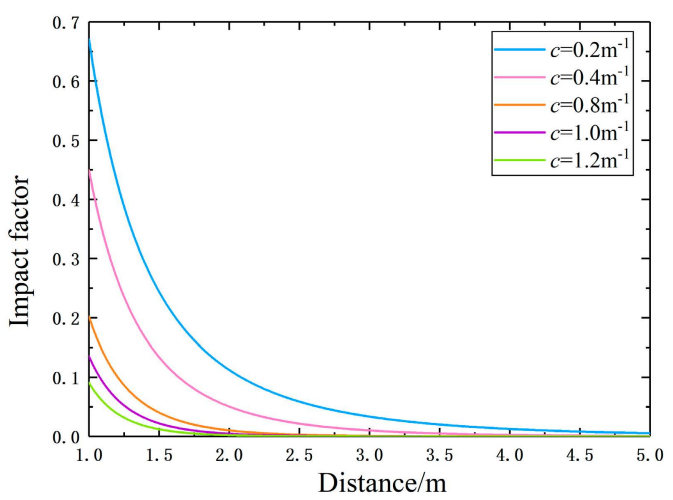

Figure 5. Impact factor variation with $R$ for five different $c$ values.

Having analyzed the influence of distance, we analyze attenuation coefficient in this section. Distance $R$ is fixed for five different constant values from 1 to $5 \mathrm{~m}$. The effect of the attenuation coefficient is analyzed in Figure 6. The decrementing of the impact factor can be seen with the rise in target distance and medium attenuation coefficient. When the attenuation coefficient is equal to $1,20 \%$ of the light can transmitted after $1 \mathrm{~m}$, which shows that the attenuation coefficient has a strong effect on the light's propagation.

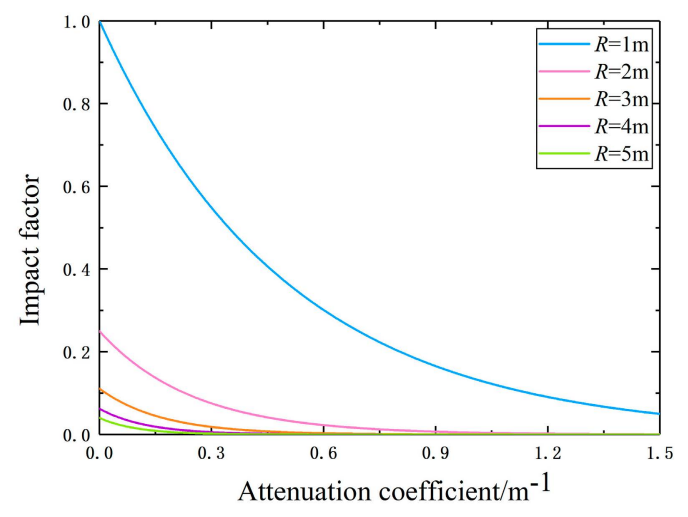

Figure 6. Impact factor variation with $c$ with for five different $R$ values.

According to analysis of various parameters above, it can be concluded that the reflected light intensity goes down with increments in the angle of incidence, distance, and medium attenuation coefficient, will further cause increments in the intensity error. Hence, to get better results, it is necessary to control these factors to be as small as possible in real applications.

\section{Experimental Investigation}

For the underwater SPI, in order to investigate the influence of the angle of incidence, target distance and the medium attenuation on the SPI, we designed an underwater, active-mode SPI system, as shown in the schematic, Figure 7, in which the target angle, distance, and water environment can be controlled separately.

In our experiment, the angle of incidence of the projected pattern is able to be controlled by the turntable below the object, and the relative position of the collecting lens and the single-pixel detector is fixed and mounted on the guide rail for the purpose of controlling the detector and the target distance. Digital light processing (DLP) was employed in our investigation. It is a set of chipsets based on optical micro-electro-mechanical technology that uses a DMD. 


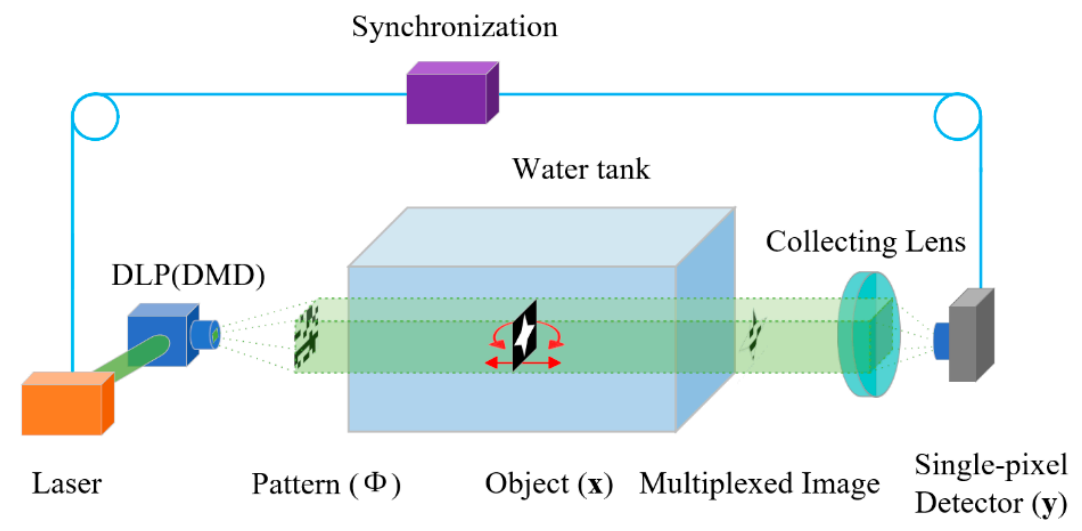

Figure 7. Experimental setups for SPI from different view angles.

For more intuitive and quantitative image quality evaluations, the modulation transfer function (MTF) measurement target pattern (proportion 1:1) was designed as shown in Figure 8. The test target pattern conforms to the MIL-STD-150A standard. Each element consists of six lines, three horizontal ones, and three vertical ones. The line-to-line spacing of each element is the same as its own width and the aspect ratio is 5:1. The six elements provide six spatial resolutions of $0.1,0.2,0.3,0.4,0.5$, and 0.6 $\mathrm{lp} / \mathrm{mm}$. The resolution is determined by the observation system's imaging resolution of the horizontal and vertical lines in the measurement target, wherein the maximum indistinguishable horizontal and vertical lines determine the ultimate resolution of the imaging system.

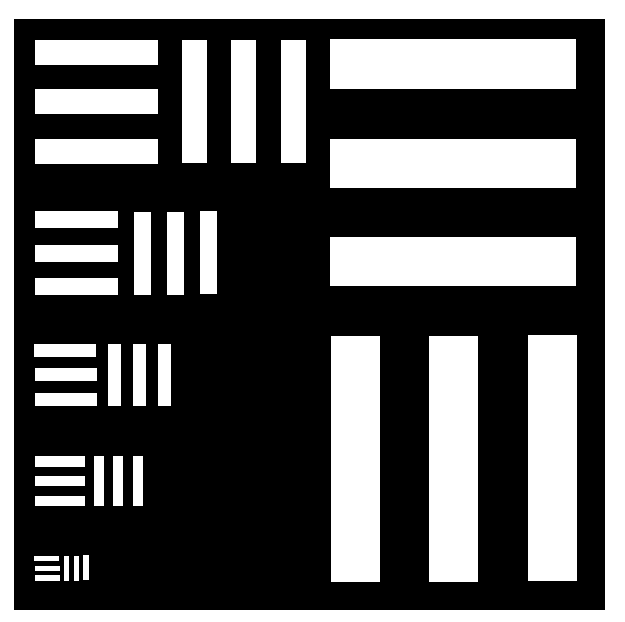

Figure 8. Resolution test targets pattern.

\section{Results and Analysis}

\subsection{Influence of Angle of Incidence}

For the angle of incidence influence on the SPI, Figure 7 shows the experiment apparatus for the angle of view (AOV) of SPI, where the positions of light source and the detector are kept unchanged and the target position is changed on the turntable to get imaging results from different viewing angles.

In the current study, we chose a two-dimensional reflective target and set the target at $0^{\circ}, 40^{\circ}$, and $60^{\circ}$, at four different positions. The results captured from different angles are shown in Figure 9. From the three images in Figure 9, we can clearly see that with an increase of the angle of incidence, the reconstructed results captured by the SPI system become more degraded, which is due to the fact that the reflected light intensity becomes more unstable as the angle of incidence increases. As the angle of incidence increases, the contrast between the central region and the background region of 
the reconstructed image gradually decreases, the overall contrast of the image decreases, and the signal-to-noise ratio of the reconstructed image decreases.

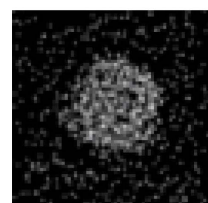

(a)

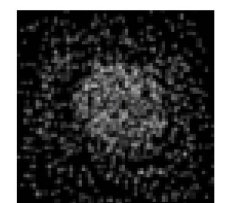

(b)

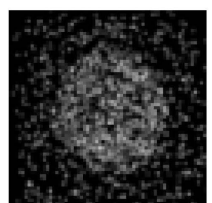

(c)

Figure 9. Experimental results of setup with different angles. (a) $0^{\circ}$; (b) $40^{\circ}$; (c) $60^{\circ}$.

The test target pattern was printed on a 1:1 ratio using a UV printer on three target substrates of flat carbon steel, polished glazed tiles, and smooth cardboard. In order to ensure the reliability of the imaging quality comparison experiment, each target was fixed on the V-shaped holder, and the observation pattern generated by the Hadamard measurement matrix with a resolution of $128 \times 128$ was sequentially subjected to 5500 sampling observations for the three targets. The length of a single sub-cell projected onto the surface of the target surface was $1 \mathrm{~mm}$, which was the same as the line width of the target element with a spatial frequency of $0.5 \mathrm{lp} / \mathrm{mm}$. After screening the sample values for target individually, each of the 5000 observations was selected to form a sampling vector, and the three target's images, shown in Figure 10, were reconstructed using the reconstruction algorithm proposed above. It can be seen from the figure that the overall imaging quality of the image of the target printed on the surface of the flat-ground steel substrate is significantly better than that of the other two, and has the highest recognition degree for the six elements with different spatial resolutions. The horizontal contrast of the three images can be clearly seen to follow a pattern. As the material's own reflectivity decreases, the system's ability to reconstruct the target is continuously reduced; that is, the contrast and replication rate of the system are gradually attenuated at a certain stripe spatial frequency.

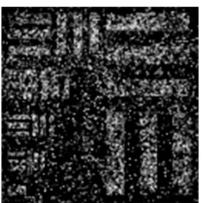

(a)

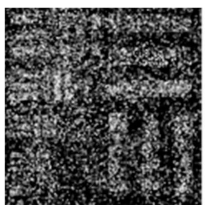

(b)

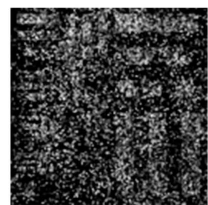

(c)

Figure 10. Reconstruction results of 5000 measurements with $128 \times 128$ resolution of the same roughness and different angles under the Hadamard pattern. (a) $0^{\circ}$; (b) $40^{\circ}$; (c) $60^{\circ}$.

Considering that the coherence of light in computational imaging has less effect on imaging quality [47], the degree of modulation of the object space information by the system may correspond to an MTF value of the system at the frequency [48]:

$$
\operatorname{MTF}(f)=100 \% \times \frac{\left(I_{\text {max }}^{\prime}-I_{\text {min }}^{\prime}\right)}{\left(I_{\text {max }}^{\prime}+I_{\text {min }}^{\prime}\right)}
$$

where $f$ is the spatial frequency of the element; $I_{\max }^{\prime}$ and $I_{\min }^{\prime}$ are the maximum and minimum gray values of the corresponding elements.

The MTF values of the six elements of the three figures in Figure 10 were calculated using Equation (16), and the three MTF curves shown in Figure 11 were plotted by interpolation. It can be seen from Figure 11 that the curve generated by fitting according to Figure 10a is significantly higher than the other two, and the trend is relatively flat in the range of spatial resolution of $0.5 \mathrm{lp} / \mathrm{mm}$ or less. However, the fitting curves generated by the three images all have a cliff-like slides when the resolution is higher than $0.5 \mathrm{lp} / \mathrm{mm}$. This is because when the spatial resolution of the object is larger than $0.5 \mathrm{lp} / \mathrm{mm}$, the side length of the observed pattern is already larger than the width of the 
object space, which exceeds the highest resolution of the system and cannot restore the object scene. The above phenomenon is basically the same as the trend shown in Figures $2-4$ above, but there is no obvious downward trend as shown in these three figures, as the angle of incidence is increasing, which also indicates to some extent that single-pixel imaging systems are insensitive to angles of incidence. Therefore, in the actual observation process, in order to improve the imaging ability of the system for high spatial frequency objects, reduce the difference between objects and images, and to improve the imaging quality, the edge of the observation pattern should be reduced as much as possible within the allowable range of the system.

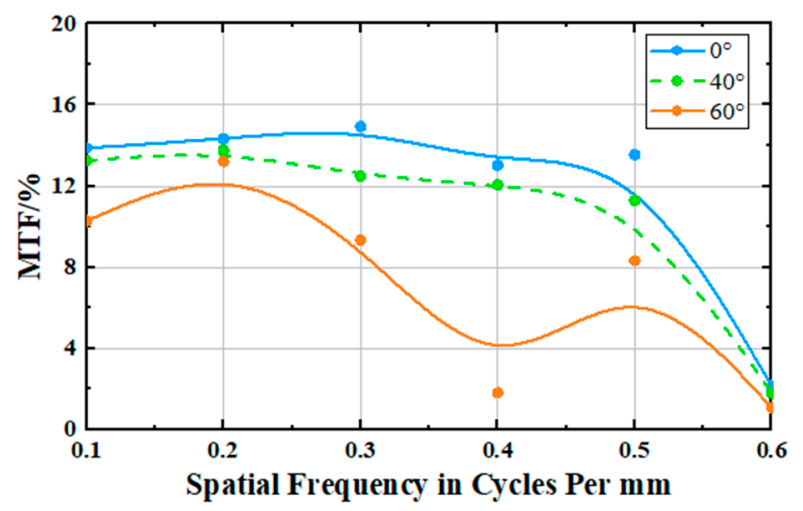

Figure 11. Modulation transfer function (MTF) fitting curve for same object at different angle.

\subsection{Influence of Target Distance}

For the distance investigation, our object was placed in four different places for the single pixel detector. Figure 12 illustrates the reconstructed results captured from different positions. From Figure 12, we can easily see that even in the case where a single-pixel imaging system is sensitive to weak light, as the target distance increases, the quality of the reconstructed image will drop dramatically. According to the four images in Figure 12, we can see that the closer objects have higher similarity to the original images, and the overall signal-to-noise ratio is higher. However, the detection results obtained for the target at a relatively long distance are poor, and the SNR ratio is relatively low.

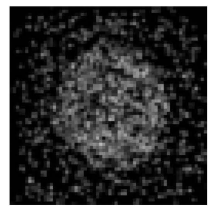

(a)

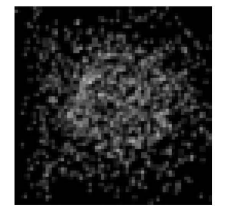

(b)

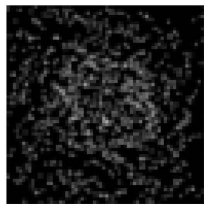

(c)

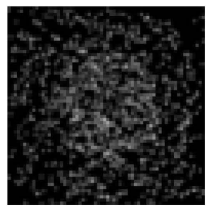

(d)

Figure 12. Experimental results of setup with different distancse: (a) $1 \mathrm{~m}$; (b) $2 \mathrm{~m}$; (c) $3 \mathrm{~m}$; (d) $4 \mathrm{~m}$.

In the target distance section, in order to further compare the analytical ability of the system to the non-positional target and the image reconstruction quality in each case, the target image was printed on a rectangular, parallelepiped carbon steel surface with a surface roughness of $0.8 \mu \mathrm{m}$ using a UV printer. The active-mode, single-pixel imaging system performed 5500 sampling observation experiments on four targets, and selected 5000 observations in each group and reconstructed them using the reconstruction algorithm proposed in this paper, as shown in Figure 13a-d. Among the four images, the black and white two-color target at the one-meter position has the highest image sharpness, and the target image obtained at the four-meter position has the worst image quality, which is consistent with the reflectivity characteristics discussed above. 


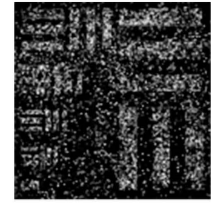

(a)

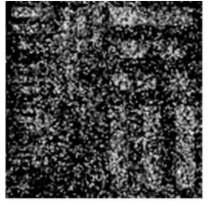

(b)

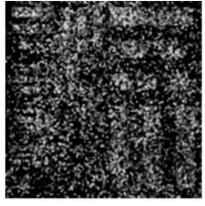

(c)

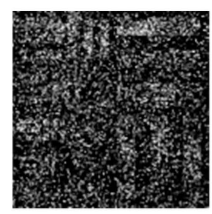

(d)

Figure 13. Reconstruction results of 5000 measurements with $128 \times 128$ resolution of the same roughness and different distances under the Hadamard pattern: (a) 1 m; (b) 2 m; (c) 3 m; (d) 4 m.

According to Equation (16) and the four images obtained, the MTF values and the fitted MTF curves under the corresponding elements in the figure are plotted in Figure 13. Comparing the four MTF curves, it can be clearly seen that when the target is near from the system, the area of the package below the MTF curve will be larger. When the spatial resolution is lower than $0.5 \mathrm{lp} / \mathrm{mm}$, the MTF curves corresponding to the four colors are relatively smooth, indicating that the consistency of the edge and the center is higher in the reconstructed image, the aberration control of the system is ideal, and the overall imaging quality is better. The MTF curve shows a relatively significant downward trend only when the spatial resolution is higher than $0.5 \mathrm{lp} / \mathrm{mm}$. Comparing the results shown in Figures 13 and 14, we can clearly see that as the target distance increases, the value of the influence factor will be greatly reduced, and the imaging quality of the target at the corresponding distance will also decrease.

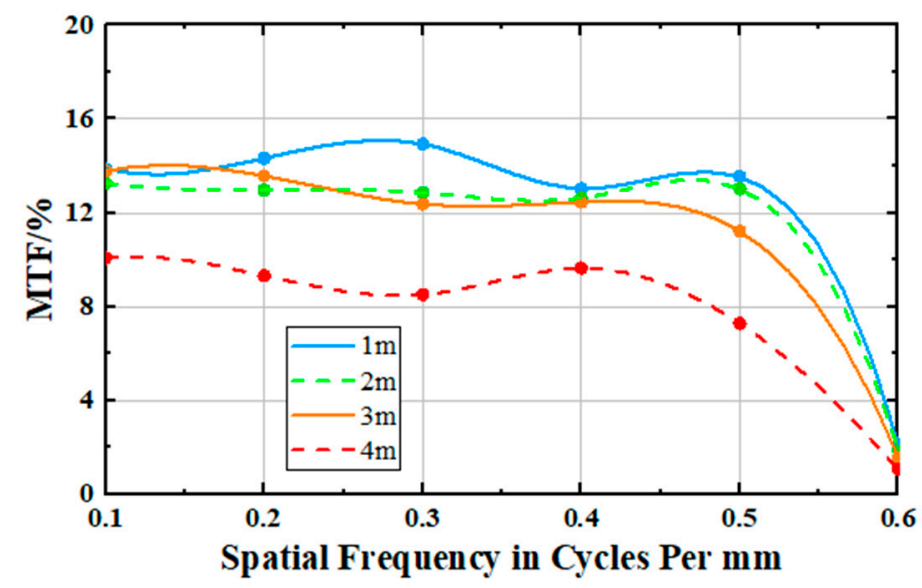

Figure 14. MTF fitting curve for same object at different distances.

\subsection{Influence of Medium Attenuation}

At the end, the influence of attenuation coefficient caused by the absorption and backscattering of propagation medium will be discussed. In the simulation, it was shown that the attenuation coefficient has a strong impact on reflectance. To get maximum reflected light, the target was placed one-meter away from the laser source with angle of incidence: zero. Chinese clay was used to create an underwater condition set with a different degree of turbidity. By controlling the amount of the Chinese clay, the range of attenuation coefficients of the water became $1.1 \mathrm{~m}^{-1}-1.4 \mathrm{~m}^{-1}$ which was measured by using a light transmittance meter. The attenuation coefficient of medium is reciprocal to the readings on the light transmittance meter:

$$
c=\frac{1}{L T}
$$

in which $c$ is the medium attenuation coefficient and $L T$ is the light transmissivity given by the transmittance meter.

When light penetrates the water, photons are either scattered or absorbed. While scattering redirects the angle of the photon path, absorption removes the photons permanently from the 
path. Unlike scattering, which is weakly spectrally dependent, absorption is highly spectrally dependent. The magnitude and spectral features depend upon the concentration and composition of the particulate and dissolved constituents and water itself. The IOPs (inherent optical properties) are conservative properties, and therefore, the magnitude of the absorption coefficient varies linearly with the concentration of the absorbing material $[49,50]$. Therefore, as the concentration of the medium continues to increase, the attenuation factor of the medium is gradually increased, and the effective light intensity that can reach the receiver is continuously reduced, thereby affecting the overall quality of the system.

In this part of our study, we focus on the influence of medium attenuation, which can be considered the turbidity. In order to simulate the environmental turbidity, we put our reflective target in a water tank, and the single detector just outside the water tank. Figure 15 shows the recovered images in different turbidity levels. Comparing the four images, it can be clearly seen that the boundary of the target becomes more and more inconspicuous, and the background noise is significantly increased, which is consistent with the theoretical analysis.

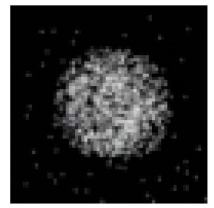

(a)

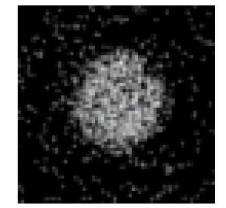

(b)

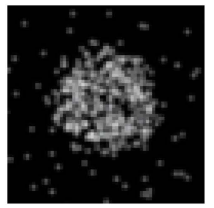

(c)

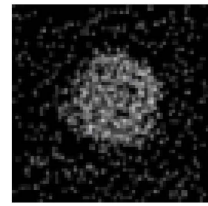

(d)

Figure 15. Experimental results of setup with different turbid degrees: $(\mathbf{a}) c=1.1 \mathrm{~m}^{-1}$; (b) $c=1.2 \mathrm{~m}^{-1}$; (c) $c=1.3 \mathrm{~m}^{-1} ;$ (d) $c=1.4 \mathrm{~m}^{-1}$.

Furthermore, in order to more clearly reflect the correspondence between image quality and medium attenuation, the target pattern shown in Figure 8 is printed on a flat-ground carbon steel surface having a surface roughness of $0.8 \mu \mathrm{m}$ and being of a ratio of 1:1. The medium attenuation is controlled by $c=1.1 \mathrm{~m}^{-1}, c=1.2 \mathrm{~m}^{-1}, c=1.3 \mathrm{~m}^{-1}$, and $c=1.4 \mathrm{~m}^{-1}$ conditions. Using the system in this chapter, 5500 sampling observations were performed on the four targets, and four groups of 5000 observations were selected to form a sampling matrix. The four images shown in Figure 16 were reconstructed by Hadamard sampling matrices and the reconstruction algorithm of this paper. It can be clearly seen from the four images that as the attenuation of the medium decreases, the sharpness of the target image obtained by the reconstruction gradually increases, the edges of the lines in each element become clearer, and the blurred image of the reconstructed image becomes smoother and more layered.

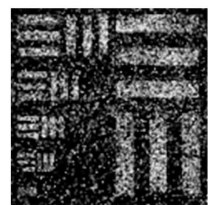

(a)

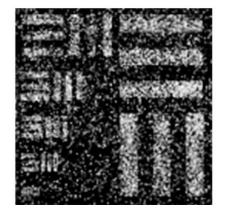

(b)

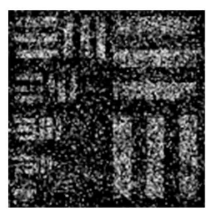

(c)

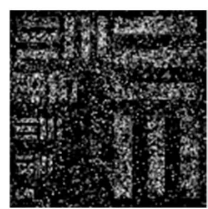

(d)

Figure 16. Reconstruction results of 5000 measurements with $128 \times 128$ resolution of the different medium attenuations under the Hadamard pattern: $(\mathbf{a}) c=1.1 \mathrm{~m}^{-1} ;(\mathbf{b}) c=1.2 \mathrm{~m}^{-1} ;$ (c) $c=1.3 \mathrm{~m}^{-1}$; (d) $c=1.4 \mathrm{~m}^{-1}$.

Using the same method, the MTF fit curves of four different media turbidities, as shown in Figure 17, were plotted based on the four reconstructed images. It can be seen from the four images in Figure 16 and the four curves in Figure 17 that the lower the turbidity of the medium, the larger the integral value of the MTF value and the spatial resolution, the better the system's optical quality. Besides, within the range of system resolution limits, the four MTF curves are relatively stable, indicating that the image quality of the corner region and the central region in the reconstructed image is not obvious, 
the overall resolution of the system is high, and the difference between the reconstructed image and the real scene of the object is small. Combined with the trend of the influence factor shown in Figures 5 and 6 with the environmental attenuation, it can be seen that when the attenuation of the environment increases, the influence factor value will decrease significantly. This apparent downward trend has exceeded noise tolerance for general conventional imaging systems, but has not had a significant impact on our SPI systems.

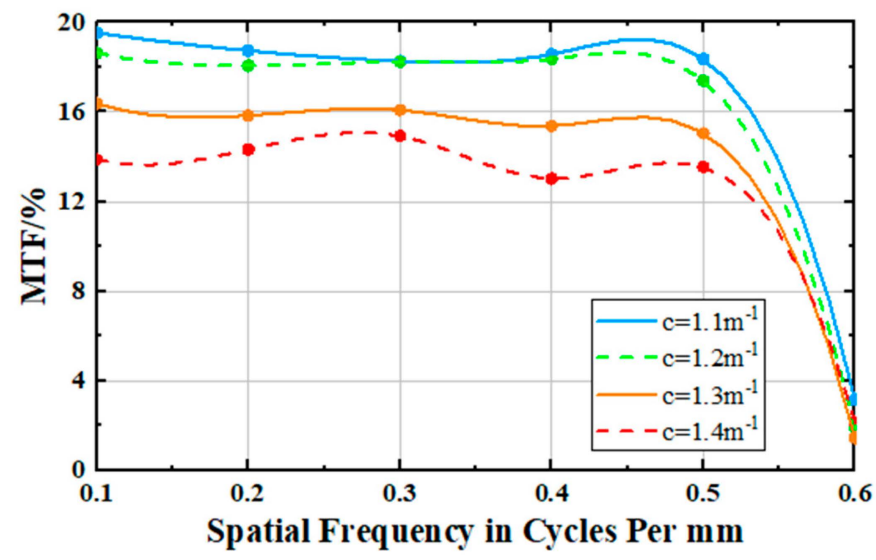

Figure 17. MTF fitting curve for different medium attenuation.

\section{Conclusions}

We investigated the influence of angle of incidence, target distance, and medium attenuation on an underwater SPI system using a theoretical and experimental study. Using BRDF and LADAR models, a theoretical deviation was conducted to obtain the dependencies of reflectance due to angle of incidence, target distance, and medium attenuation coefficient. The variation in intensity is crucial, as it directly impacts the performance of system.

Three experimental condition parameters, namely, angle of incidence, target distance, and medium attenuation, were analyzed to determine their effects on the image reconstruction quality and the system resolution level. Theoretical analysis reveals that intensity accuracy and the SPI system detection capability directly depends on reflection ratio and ultimately relies on the parameters investigated. The intensity accuracy increases when these parameters decrease and adheres to the reflection model used. Our experimental results matched the theoretical analysis well. When the angle of incidence increases from $0^{\circ}$ to $60^{\circ}$, the MTF value decreases from 0.147 to 0.019 at $0.3 \mathrm{lp} / \mathrm{mm}$; when the target distance is gradually increased from 1 to $4 \mathrm{~m}$, the MTF value decreases from 0.147 to 0.082 at $0.3 \mathrm{~mm} / \mathrm{lp}$; when the attenuation of the environment increases from $1.1 \mathrm{~m}$ to $1.4 \mathrm{~m}^{-1}$, the MTF value decreases from 0.182 to 0.141 at $0.3 \mathrm{~mm} / \mathrm{lp}$. The above experimental results are in good agreement with the theoretical analysis in the paper, which proves the correctness of theory and experiment.

Our contributions can be used for some applications, such as target recognition, object modelling, and laser tracking systems. It should be also useful for imaging applications, such as ghost imaging and a range gated system where objective work condition characteristics are required. In future, to extend our study on underwater ranging system, we will investigate other factors, such as laser wavelength and target characteristics. We will also look into a solution for overcoming the influence of objective work conditions we have discussed in this paper.

Author Contributions: This research work was mainly conducted by Q.C. at Monash University Malaysia. He composed manuscript. A.M. contributed to the experimental investigation and results analysis. X.X. is Chen's supervisor and contributed to manuscript revising. X.W. is Chen's external supervisor, and contributed to the system's design, the results' analysis, and the manuscript revising.

Funding: This work was supported in part by the Ministry of Higher Education, Malaysia under grant FRGS/1/2016/STG02/ MUSM/02/1. 
Conflicts of Interest: The authors declare no conflict of interest.

\section{References}

1. Wang, N.; Zheng, B.; Zheng, H.; Yu, Z. Feeble object detection of underwater images through LSR with delay loop. Opt. Express 2017, 25, 22490-22498. [CrossRef]

2. Sivčev, S.; Rossi, M.; Coleman, J.; Omerdić, E.; Dooly, G.; Toal, D. Collision Detection for Underwater ROV Manipulator Systems. Sensors 2018, 18, 1117. [CrossRef] [PubMed]

3. Le, M.; Wang, G.; Zheng, H.; Liu, J.; Zhou, Y.; Xu, Z. Underwater computational ghost imaging. Opt. Express 2017, 25, 22859-22868. [CrossRef] [PubMed]

4. Han, P.L.; Liu, F.; Zhang, G.; Tao, Y.; Shao, X.P. Multi-scale analysis method of underwater polarization imaging. Acta Phys. Sin. 2018, 67, 054202.

5. Gholami, A.; Saghafifar, H. Simulation of an active underwater imaging through a wavy sea surface. J. Mod. Opt. 2018, 65, 1210-1218. [CrossRef]

6. Wu, T.C.; Chi, Y.C.; Wang, H.Y.; Tsai, C.T.; Lin, G.R. Blue Laser Diode Enables Underwater Communication at 12.4 Gbps. Sci. Rep. 2017, 7, 40480. [CrossRef]

7. Vidvans, A.; Basu, S. Smartphone based scalable reverse engineering by digital image correlation. Opt. Lasers Eng. 2018, 102, 126-135. [CrossRef]

8. Lee, J.; Lee, K.; Lee, S.; Kim, S.W.; Kim, Y.J. High precision laser ranging by time-of-flight measurement of femtosecond pulses. Meas. Sci. Technol. 2012, 23, 207. [CrossRef]

9. Amann, M.C.; Myllylae, R.A. Laser ranging: A critical review of unusual techniques for distance measurement. Opt. Eng. 2001, 40, 10-19.

10. Huke, P.; Klattenhoff, R.; Von Kopylow, C.; Bergmann, R.B. Novel Trends in Optical Non-Destructive Testing Methods. J. Eur. Opt. Soc. Rapid Publ. 2011, 8, 1672-1675. [CrossRef]

11. Lv, D.; Sun, J.F.; Li, Q.; Wang, Q. Model-based recognition of 3D articulated target using ladar range data. Appl. Opt. 2015, 54, 5382-5391. [CrossRef] [PubMed]

12. Baumann, E.; Giorgetta, F.R.; Deschênes, J.D.; Swann, W.C.; Coddington, I.; Newbury, N.R. Comb-calibrated laser ranging for three-dimensional surface profiling with micrometer-level precision at a distance. Opt. Express 2014, 22, 24914-24928. [CrossRef] [PubMed]

13. Mccarthy, A.; Collins, R.J.; Krichel, N.J.; Fernández, V.; Wallace, A.M.; Buller, G.S. Long-range time-of-flight scanning sensor based on high-speed time-correlated single-photon counting. Appl. Opt. 2009, 48, 6241-6251. [CrossRef] [PubMed]

14. Andersson, P. Long-range three-dimensional imaging using range-gated laser radar images. Opt. Eng. 2006, 45, 34301. [CrossRef]

15. Zevallos, L.; Manuel, E.; Gayen, S.K.; Alrubaiee, M.; Alfano, R.R. Time-gated backscattered ballistic light imaging of objects in turbid water. Appl. Phys. Lett. 2005, 86, 011113-011115. [CrossRef]

16. Sluzek, A.; Chai, T.Y.; Chen, Y.F.; Tan, C.S.; Seet, G.; Wong, H.Y.; Xin, W. Scattering noise estimation of range-gated imaging system in turbid condition. Opt. Express 2010, 18, 21147-21154.

17. Mullen, L.; Cochenour, B. Optical modulation techniques for underwater detection, ranging and imaging. Proc. SPIE. 2011, 8030. [CrossRef]

18. Du, B.; Pang, C.; Wu, D.; Li, Z.; Peng, H.; Tao, Y.; Wu, E.; Wu, G. High-speed photon-counting laser ranging for broad range of distances. Sci. Rep. 2018, 8, 4198. [CrossRef]

19. Gao, J.; Sun, J.; Wei, J.; Wang, Q. Research of underwater target detection using a Slit Streak Tube Imaging Lidar. In Proceedings of the Academic International Symposium on Optoelectronics and Microelectronics Technology, Harbin, China, 12-16 October 2011; pp. 240-243.

20. Tan, C.; Sluzek, A.; Seet, G.; Shacklock, A. Three-dimensional Machine Vision Using Gated Imaging System: A Numerical Analysis. In Proceedings of the 2006 IEEE Conference on Robotics, Automation and Mechatronics, Bangkok, Thailand, 1-3 June 2006; pp. 1-6.

21. Höfle, B.; Pfeifer, N. Correction of laser scanning intensity data: Data and model-driven approaches. ISPRS J. Photogramm. Remote Sens. 2007, 62, 415-433. [CrossRef]

22. Chua, S.Y.; Wang, X.; Guo, N.; Tan, C.S. Range compensation for accurate 3D imaging system. Appl. Opt. 2016, 55, 153-158. [CrossRef] 
23. Wang, X.; Zhou, Y.; Liu, Y. Impact of echo broadening ef fect on active range-gated imaging. Chin. Opt. Lett. 2012, 10, 32-34.

24. Xinwei, W.; Youfu, L.; Yan, Z. Triangular-range-intensity profile spatial-correlation method for 3D super-resolution range-gated imaging. Appl. Opt. 2013, 52, 7399-7406. [CrossRef] [PubMed]

25. Yang, K. Analysis of MCP gain selection for underwater range-gated imaging applications based on ICCD. J. Mod. Opt. 2010, 57, 408-417.

26. Wang, X.; Hu, L.; Zhi, Q.; Chen, Z.; Jin, W. Influence of range-gated intensifiers on underwater imaging system SNR. In Proceedings of the International Symposium on Photoelectronic Detection \& Imaging, Beijing, China, 25-27 June 2013; p. 89120.

27. Laux, A.; Jantzi, A.; Cochenour, B.; Mullen, L.; Jemison, W. Enhanced underwater ranging using an optical vortex. Opt. Express 2018, 26, 2668-2674.

28. Zheng, B.; Wang, N.; Zheng, H.; Yu, Z.; Wang, J. Object extraction from underwater images through logical stochastic resonance. Opt. Lett. 2016, 41, 4967-4970. [CrossRef] [PubMed]

29. Xu, J.; Song, Y.; Yu, X.; Lin, A.; Kong, M.; Han, J.; Deng, N. Underwater wireless transmission of high-speed QAM-OFDM signals using a compact red-light laser. Opt. Express 2016, 24, 8097-8109. [CrossRef] [PubMed]

30. Hung, C.C.; Tsao, S.C.; Huang, K.H.; Jang, J.P.; Chang, H.K.; Dobbs, F.C. A highly sensitive underwater video system for use in turbid aquaculture ponds. Sci. Rep. 2016, 6, 31810. [CrossRef]

31. Britton, W.B.; Dalgleish, F.R.; Zhuang, H. Beamforming receiver for underwater pulsed laser line scanning. In Proceedings of the Ocean Sensing and Monitoring X., Orlando, FL, USA, 25 May 2018; p. 106310.

32. Duan, Y.; Song, C. Influence of Atmospheric Aerosol Backscattering on Incoherent Frequency Modulation Continuous-wave Laser Ranging in the Fog. Eng. Lett. 2017, 25, 15-21.

33. Chen, Q.; Chamoli, S.K.; Yin, P.; Wang, X.; Xu, X. Imaging of hidden object using passive mode single pixel imaging with compressive sensing. Laser Phys. Lett. 2018, 15, 126201. [CrossRef]

34. Lee, J.; Kim, Y.J.; Lee, K.; Lee, S.; Kim, S.W. Time-of-flight measurement with femtosecond light pulses. Nat. Photonics 2010, 4, 207. [CrossRef]

35. Bender, P.L.; Currie, D.G.; Poultney, S.K.; Alley, C.O.; Dicke, R.H.; Wilkinson, D.T.; Eckhardt, D.H.; Faller, J.E.; Kaula, W.M.; Mulholland, J.D. The Lunar Laser Ranging Experiment: Accurate ranges have given a large improvement in the lunar orbit and new selenophysical information. Science 1973, 182, 229-238. [CrossRef] [PubMed]

36. Friedland, S.; Li, Q.; Dan, S.; Bernal, E.A. Two Algorithms for Compressed Sensing of Sparse Tensors; Springer International Publishing: Berlin, Germany, 2015; pp. 259-281.

37. Min, S.O.; Hong, J.K.; Kim, T.H.; Hong, K.H.; Kim, B.W.; Dong, J.P. Time-of-Flight Analysis of Three-Dimensional Imaging Laser Radar Using A Geiger-Mode Avalanche Photodiode. Jpn. J. Appl. Phys. 2010, 49, 026601-026606.

38. Nicodemus, F.E. Directional Reflectance and Emissivity of an Opaque Surface. Appl. Opt. 1965, 4, 767-773. [CrossRef]

39. Walter, B.; Marschner, S.R.; Li, H.; Torrance, K.E. Microfacet Models for Refraction through Rough Surfaces. In Proceedings of the Eurographics Symposium on Rendering Techniques, Grenoble, France, 25-27 June 2007; pp. 195-206.

40. Ashikmin, M.; Shirley, P. A microfacet-based BRDF generator. In Proceedings of the Conference on Computer Graphics and Interactive Techniques, New York, NY, USA, 23-28 July 2000; pp. 65-74.

41. Schlick, C. An Inexpensive BRDF Model for Physically-based Rendering. Comput. Graph. Forum 1994, 13, 233-246.

42. Sun, J.; Wang, Q. 4-D image reconstruction for Streak Tube Imaging Lidar. Laser Phys. 2009, 19, 502. [CrossRef]

43. Nevis, A.J. Automated processing for streak tube imaging lidar data. In Proceedings of the SPIE-The International Society for Optical Engineering, Orlando, FL, USA, 24-25 April 2003; p. 5089.

44. Gelbart, A. Flash lidar based on multiple-slit streak tube imaging lidar. In Proceedings of the Laser Radar Technology and Applications VII, Orlando, FL, USA, 3-4 April 2002; pp. 9-18.

45. Busck, J.; Heiselberg, H. Gated viewing and high-accuracy three-dimensional laser radar. Appl. Opt. 2004, 43, 4705-4710. [CrossRef]

46. Seubert, C.M.; Nichols, M.E.; Frey, J.; Shtein, M.; Thouless, M.D. The characterization and effects of microstructure on the appearance of platelet-polymer composite coatings. J. Mater. Sci. 2016, 51, 2259-2273. [CrossRef] 
47. Field, J.J.; Wernsing, K.A.; Squier, J.A.; Bartels, R.A.J.J.A. Three-dimensional single-pixel imaging of incoherent light with spatiotemporally modulated illumination. J. Opt. Soc. Am. A 2018, 35, 1438-1449. [CrossRef]

48. Huang, X.; Netravali, R.; Man, H.; Lawrence, V.J.S. Multi-sensor fusion of infrared and electro-optic signals for high resolution night images. Sensors 2012, 12, 10326-10338. [CrossRef]

49. Dolin, L.S.J.A.O. Theory of lidar method for measurement of the modulation transfer function of water layers. Appl. Opt. 2013, 52, 199-207. [CrossRef]

50. Mobley, C.; Boss, E.; Roesler, C. Ocean Optics Web Book. Available online: http://www.oceanopticsbook.info/ (accessed on 11 November 2019).

C 2019 by the authors. Licensee MDPI, Basel, Switzerland. This article is an open access article distributed under the terms and conditions of the Creative Commons Attribution (CC BY) license (http://creativecommons.org/licenses/by/4.0/). 